\title{
Accurate Bit Error Rate Calculation for Asynchronous Chaos-Based DS-CDMA over Multipath Channel
}

\author{
Georges Kaddoum,, ${ }^{1,2}$ Daniel Roviras, ${ }^{3}$ Pascal Chargé, ${ }^{2}$ and Daniele Fournier-Prunaret ${ }^{2}$ \\ ${ }^{1}$ IRIT Laboratory, University of Toulouse, 2 rue Charles Camichel, 31071 Toulouse cedex, France \\ ${ }^{2}$ LATTIS Laboratory, University of Toulouse, 135 avenue de Rangueil, 31077 Toulouse cedex 4, France \\ ${ }^{3}$ LAETITIA Laboratory, CNAM Paris, 292 rue Saint-Martin, 75003 Paris, France
}

Correspondence should be addressed to Georges Kaddoum, gkaddoum@enseeiht.fr

Received 21 January 2009; Revised 26 June 2009; Accepted 23 July 2009

Recommended by Kutluyil Dogancay

\begin{abstract}
An accurate approach to compute the bit error rate expression for multiuser chaosbased DS-CDMA system is presented in this paper. For more realistic communication system a slow fading multipath channel is considered. A simple RAKE receiver structure is considered. Based on the bit energy distribution, this approach compared to others computation methods existing in literature gives accurate results with low computation charge. Perfect estimation of the channel coefficients with the associated delays and chaos synchronization is assumed. The bit error rate is derived in terms of the bit energy distribution, the number of paths, the noise variance, and the number of users. Results are illustrated by theoretical calculations and numerical simulations which point out the accuracy of our approach.
\end{abstract}

Copyright (c) 2009 Georges Kaddoum et al. This is an open access article distributed under the Creative Commons Attribution License, which permits unrestricted use, distribution, and reproduction in any medium, provided the original work is properly cited.

\section{Introduction}

Communication using chaos has attracted a great deal of attention from many researchers for more than a decade. Motivations of these studies remain to the advantages that are offered by chaotic signals such as robustness in multipath environments, resistance to jamming [1]. Chaotic signals are non periodic, broadband, and difficult to predict and to reconstruct. These are properties which coincide with requirements for signal used in communication systems, in particular for spread-spectrum communications and secure communications $[1,2]$.

Many communication systems were inspired by the synchronization results of Pecora and Carrol [3], focused on analog modulation schemes with coherent receivers [47]. Digital modulations using discrete signals and a coherent receiver were introduced in [8]. Many others chaotic digital modulation schemes were proposed and studied [9-11]. It has been found that digital schemes are comparatively more robust than analog schemes in the presence of noise and thus represent a more practical form of systems for implementation. Direct application of chaos to conventional direct-sequence spread-spectrum (DSSS) systems was also reported on the code level $[12,13]$. The basic principle is to replace the conventional binary spreading sequences, such as m-sequences or Gold sequences [14], by the chaotic sequences generated by a discrete-time nonlinear map. The advantage of using chaotic spreading sequences relies on the fact that the spreaded signal is less vulnerable to interception. Instead of applying analog chaotic sequences to spread data symbols, Mazzini et al. proposed quantizing and periodically repeating the chaotic time series for spreading. It was also reported that systems using the periodic quantized sequences have larger capacities and lower bit-error rates than those using $\mathrm{m}$-sequences and Gold sequences in a multiple-access environment $[15,16]$. A large literature exists also on chaotic spreading sequences design [17] and optimization [18-20].

Among the various digital chaos-based communication schemes, coherent chaos-shift-keying (CSK) and noncoherent differential chaos-shift-keying (DCSK) schemes have been most thoroughly analyzed [21-25]. Compared with chaotic-sequence spread-spectrum modulation, CSK and DCSK modulation schemes make use of analog chaotic wideband waveforms directly to represent the binary symbols. 
Coherent systems like CSK and chaos-based DS-CDMA require coherent correlators with the assumption that the receiver is able to generate a locally synchronous chaotic signal.

In order to compute the bit-error rate (BER) performance, many various assumptions have been presented. Because of these assumptions, computed BERs are generally different from their true value. The simplest approximation used in [26], for example, is to consider the transmitted chaotic bit energy being constant. This approximation can be reasonable when the considered spreading factors are very large (symbol duration much greater than chaotic chip duration). Nevertheless, for small or moderated spreading factors these assumption yields to very imprecise BER performance. In fact, because of the nonperiodic nature of chaotic signals, the transmitted bit energy of chaos-based DS-CDMA systems varies from one bit to another.

Another classical assumption is to use the Gaussian approximation for the decision parameter at the correlator output, $[2,20,27,28]$, by considering the sum of dependent variables as a Gaussian variable. Tam et al. in [28] have proposed a simple way of deriving the BER of the CSK system by computing numerically the first two moments of chaotic signal correlation functions. Since the real values chaotic signals are generated from a deterministic generator, the Gaussian approximation can be valid for high spreading factors but suffers from precision for small ones [29].

A mathematical calculation of BER for single and multiuser chaos communication system was recently presented by Lawrance et al. in $[29,30]$. In their approach, they did not use neither the constant bit energy approximation nor the Gaussian assumption. Only additive channel noise and multiple access interference noise follow, in their study, a Gaussian distribution. Their approach takes into account the dynamics properties of the chaotic sequence by integrating the BER expression for a given chaotic map over all possible chaotic sequences for a given spreading factor. This latter method is compared to the BER computation under Gaussian assumption in [29] and seems more realistic to match the BER. But, as it is said in [30], the aim of the method was not to give implementable procedures for realistic sized systems.

Because previous presented approaches are not valid for small spreading factors or have a higher complexity of calculation, another accurate approach was recently developed in [31-34] to compute the BER performance for single and multiuser chaos-based DS-CDMA over an Additive White Gaussian Noise (AWGN) channel. Numerical derivation of BER performance over multipath channel is also studied in [35]. The idea is to compute the Probability Density Function (PDF) of the chaotic bit energy and to integrate BER over all possible values of the PDF. The shape of the PDF bit energy is a qualitative indication concerning expected BER performances.

In this paper, an asynchronous coherent multiuser chaosbased DS-CDMA system is studied and evaluated. For each user a multipath channel is considered. Our system is quite similar to the coherent CSK system. The analog chaotic wideband waveform is used directly to spread the binary symbols. In the following we will focus our study on BER performance. For a similar studied system, the problem of performance optimization was widely studied in $[27,36]$. In our paper, we are only interested by computing the analytical performance of such a chaos-based DS-CDMA.

For a conventional DS-CDMA system under multipath channel, an RAKE receiver is used to overcome the severe consequences of the multipath fading channel. This receiver consists of a bank of programmable correlators that correlate each of the $L$ received replicas of the same transmitted signal by the corresponding locally generated code. These codes are used to despread the data signals. After the despreading process, the RAKE multiplies each replica by the corresponding estimated complex valued conjugate path gain (provided by the channel estimator) [14]. If the channel estimation is perfect, and all the paths are independent, the RAKE receiver with Maximum Ratio Combining (MRC) becomes an optimum receiver in the sense of highest signal to noise ratio [14].

In our system we assume that channel coefficients and delay estimation are perfectly known at the receiver. Furthermore we consider that the synchronization is achieved and maintained by the system proposed in [37]. Multipath propagation, correlation properties of spreading codes, and the RAKE receiver are taken into account to derive the BER expression, which is oriented to asynchronous environment. Further, the approach adopted here can be valid for many other chaotic communication systems.

This paper is organized as follows. Section 2 is dedicated to the description of the emitter structure. In Section 3 the RAKE with the demodulation process is presented. The statistical properties of multiuser and self interference noises are evaluated, and BER integral formulations are established also in Section 4. Section 5 presents the BER computation methodologies. Section 6 is dedicated to the analytical BER calculation. Section 7 reports some conclusive remarks.

\section{Model of Multiuser Chaos-Based DS-CDMA System}

In this section, the configuration of the multiuser chaosbased DS-CDMA system is presented in Figure 1.

2.1. Emitter Structure. The studied system is a chaos-based DS-CDMA system with $M$ asynchronous users. The data information symbols of user $m\left(s_{i}^{(m)}= \pm 1\right)$ with period $T_{s}$ are generated by uncorrelated sources, which are independent one from another. Symbols of user $m$ are spreaded by a chaotic sequence $x^{(m)}(t)$. Chaotic sequences of all users are generated using the same chaotic generator $f(\cdot)$ with different initial conditions. A new chaotic sample (or chip) is generated every time interval equal to $T_{c}\left(x_{k}^{(m)}=x^{(m)}\left(k T_{c}\right)\right)$ :

$$
x_{k+1}^{(m)}=f\left(x_{k}^{(m)}\right) \text {. }
$$

Chaotic sequences generated from (1) have a common mean $\mu=E(x)$ and a common variance $\sigma_{x}^{2}$. It is always possible to offset the map in order achieve $\mu=0$ without 


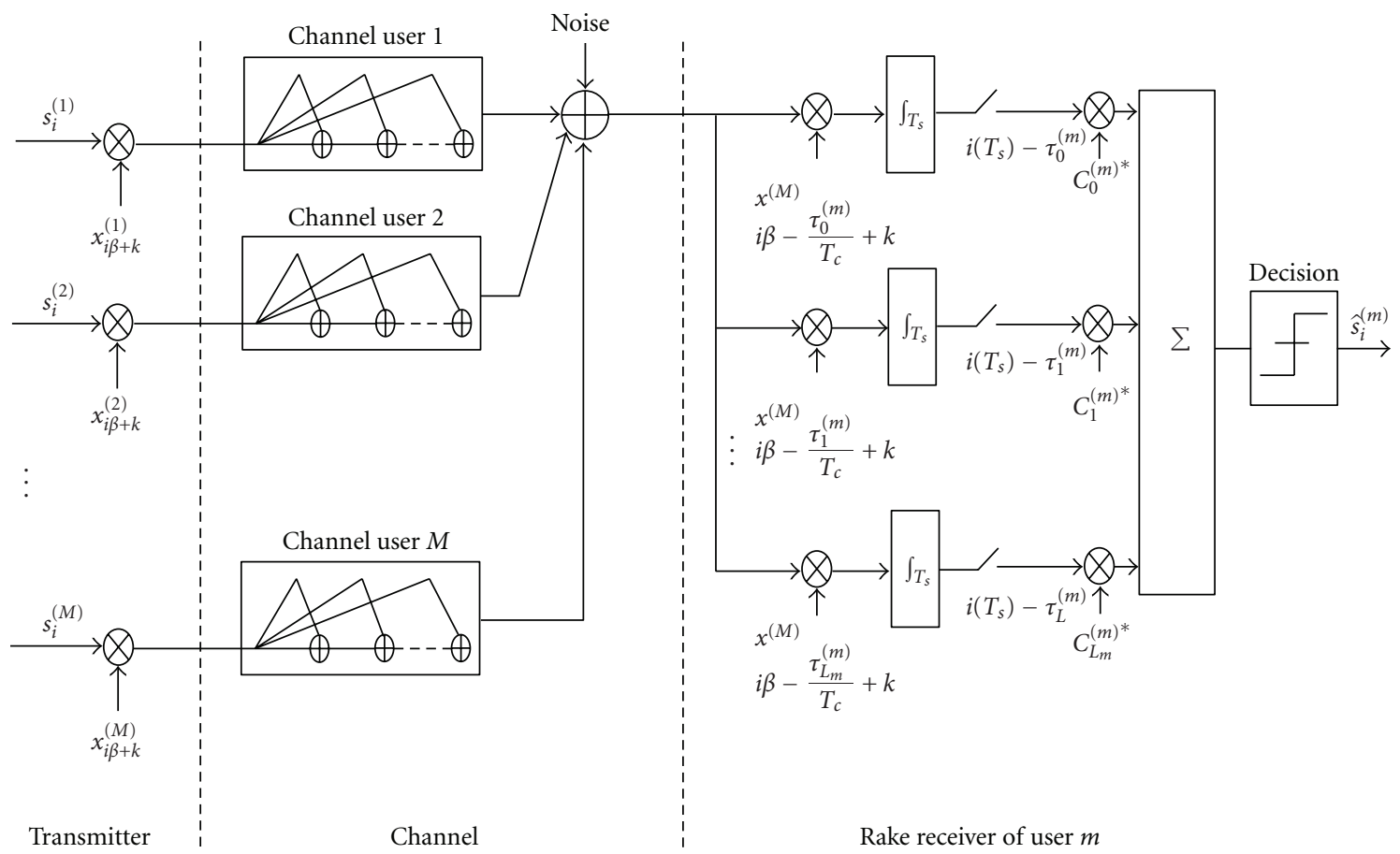

FIGURE 1: Simplified baseband equivalent of a chaos-based DS-CDMA system with a multipath channel.

changing the dynamical properties of the map. Mean values of all sequences are assumed equal to zero $(\mu=0)$ in this paper. The spreading factor $\beta$ is equal to the number of chaotic samples in a symbol duration $\left(\beta=T_{s} / T_{c}\right)$.

2.1.1. Chaotic Generator. In this paper, a Piece-Wise Linear map (PWL) is chosen as a chaotic generator sequence [38]:

$$
\left\{\begin{array}{l}
z_{k}=K\left|x_{k}\right|+\phi[\bmod 1], \\
x_{k+1}=\operatorname{sign}\left(x_{k}\right)\left(2 z_{k}-1\right) .
\end{array}\right.
$$

This map depends on $K$ and $\phi$ parameters. $K$ is a positive integer and $\phi(0<\phi<1)$ is a real number. Both can be changed to produce different sequences, and the initial condition $x(0)$ will be chosen following the condition: $0<$ $x(0)<1 / K$. In addition, this map has a known PDF for the root square of bit energy distribution. This PDF will be used later in this paper in order to derive the analytical BER expression. Without loss of generality, throughout the paper, the PWL parameters are fixed as follows: $(K=3, \phi=0.1)$.

The emitted signal of user $m$ at the output of the transmitter is

$$
u^{(m)}(t)=\sum_{i=0}^{\infty} \sum_{k=0}^{\beta-1} s_{i}^{(m)} x_{i \beta+k}^{(m)} g\left(t-(i \beta+k) T_{c}\right),
$$

where $g(t)$ is the pulse shaping filter; in this paper we have chosen a rectangular pulse of unit amplitude on $\left[0, T_{c}\right]$, that is,

$$
g(t)= \begin{cases}1, & 0 \leq t<T_{c} \\ 0, & \text { elsewhere }\end{cases}
$$

In order to simplify the mathematical model of multiuser and interuser interferences, we transformed the emitted signal into to the following form:

$$
u^{(m)}(t)=\sum_{w=0}^{+\infty} s_{\lfloor w / \beta\rfloor}^{(m)} x_{w}^{(m)} g\left(t-w T_{c}\right)
$$

where $q$ rounds the real value of $q$ to the lowest integer.

2.2. Channel Model. The channel through which the radio wave transmits are transmitted is a multipath channel. Multipath components are delayed copies of the original transmitted wave traveling through a different echo path, each with a different magnitude and time-of-arrival at the receiver. The studied system is an uplink chaos-based DSCDMA system, where each user transmits its signal over its own multipath channel.

The baseband equivalent impulse response of the normalized channel of user $m$ is

$$
h^{(m)}(\tau)=\sum_{l=0}^{L_{m}} C_{l}^{(m)} \delta\left(\tau-\tau_{l}^{(m)}\right)
$$

with

$$
\sum_{l=0}^{L_{m}}\left|C_{l}^{(m)}\right|^{2}=1
$$

where $C_{l}^{(m)}$ and $\tau_{l}^{(m)}$ denote the coefficient and the delay of the $l$ th path of user $m$ and $\delta(t)$ is the Dirac impulse. Without loss of generality delays have been taken equal to multiple values of $T_{c}$. 


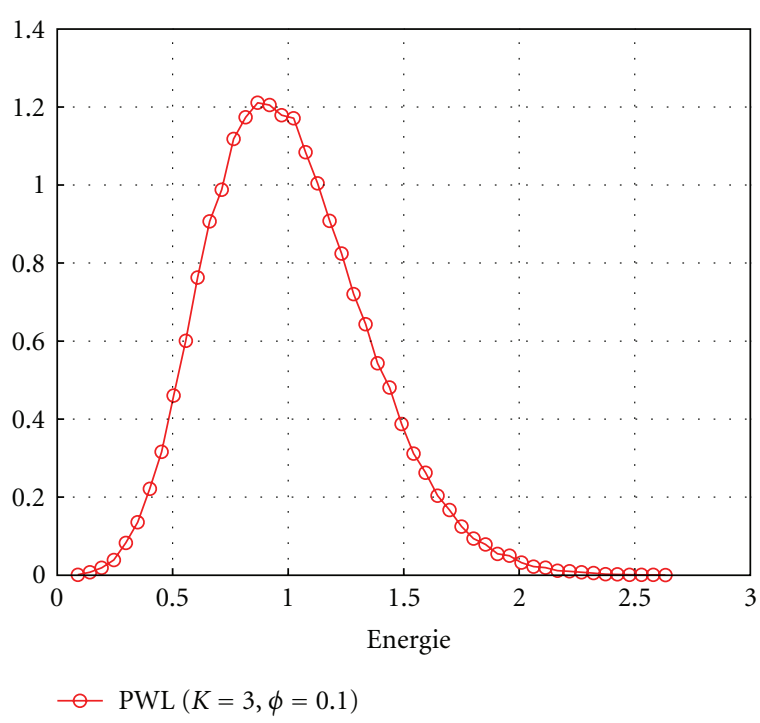

FIgURe 2: Probability density functions of bit energy for PWL chaotic sequence $(\beta=10)$.

An additive white Gaussian noise is added to the received signals. Let $n(t)$ be this noise with a two-side power spectral density given by

$$
S_{n}(f)=\frac{N_{0}}{2} .
$$

For convenience, we replace $n(t)$ by an equivalent noise source $n^{\prime}(t)$ where

$$
n^{\prime}(t)=\sum_{i=0}^{\infty} \varepsilon_{i} g\left(t-i T_{c}\right)
$$

where $\left\{\varepsilon_{i}\right\}$ is an independent Gaussian random variables with zero mean and variance:

$$
\sigma_{n}^{2}=\frac{N_{0}}{2 T_{c}} .
$$

The received signal is

$$
r(t)=\sum_{n=1}^{M} \sum_{l=0}^{L_{n}} C_{l}^{(n)} u^{(n)}\left(t-\tau_{l}^{(n)}\right)+n(t)
$$

\section{Multiuser Received Signal}

In this paper we assume that the channel estimator of the RAKE receiver estimates perfectly the delays and the associated gains. In addition, the number of channel path of each user is equal to the number of fingers at the associated receiver.

3.1. Demodulation Process. The coherent multiuser chaosbased DS-CDMA system is considered in this paper, only the user's own reference sequence is known exactly at the receiver, and no information about other user's reference sequences is known. The decision variable of user $m$ for symbol $i$ can be modelled by the sum of interest variable $Z_{i}^{(m)}$ and the different noise sources $\left(\zeta_{i}^{(m)}+\psi_{i}^{(m)}\right)$ :

$$
D_{i}^{(m)}=Z_{i}^{(m)}+\zeta_{i}^{(m)}+\psi_{i}^{(m)} .
$$

The interest variable expression $Z_{i}^{(m)}$ is

$$
Z_{i}^{(m)}=T_{c} \sum_{l=0}^{L_{m}} s_{i}^{(m)}\left|C_{l}^{(m)}\right|^{2} \sum_{k=0}^{\beta-1}\left(x_{i \beta+k}^{(m)}\right)^{2} .
$$

$E_{b c}^{(i, m)}=T_{c} \sum_{k=0}^{\beta-1}\left(x_{i \beta+k}^{(m)}\right)^{2}$ is the energy transmitted by user $m$ during its $i$ th data symbol duration.

$Z_{i}^{(m)}$ carries the information bit to be retrieved. For normalized channel coefficients the variable of interest is:

$$
Z_{i}^{(m)}=E_{b c}^{(i, m)} s_{i}^{(m)} .
$$

The expression of the AWGN noise coming from the channel after correlation with the chaotic sequence is

$$
\zeta_{i}^{(m)}=T_{c} \sum_{r=0}^{L_{m}} C_{r}^{(m) *} \sum_{k=0}^{\beta-1} x_{i \beta+k}^{(m)} n_{i \beta+k+\tau_{r}^{(m)} / T_{c}} .
$$

The multiuser and self interference noise expressions are

$$
\psi_{i}^{(m)}=\mathrm{MUI}_{i}^{(m)}+\mathrm{SI}_{i}^{(m)},
$$

$$
\psi_{i}^{(m)}=T_{c} \sum_{r=0}^{L_{m}} C_{r}^{(m) *} \sum_{w=i \beta+k}^{i \beta+\beta-1} x_{w}^{(m)}\left[\gamma_{w, i}^{(m)}+\alpha_{w, i}^{(m)}\right]
$$

where

$$
\begin{gathered}
\gamma_{w, i}^{(m)}=\sum_{\substack{n=1 \\
n \neq m}}^{M} \sum_{l=0}^{L_{n}} C_{l}^{(n)} s_{\left\lfloor\left(w+\left(\tau_{r}^{(m)}-\tau_{l}^{(n)}\right) / T_{c}\right) / \beta\right]^{(n)}}^{x_{w+\left(\tau_{r}^{(m)}-\tau_{l}^{(n)}\right) / T_{c}}^{(n)},} \\
\alpha_{w, i}^{(m)}=\sum_{\substack{l=0 \\
l \neq r}}^{L_{m}} C_{l}^{(m)} s_{\left\lfloor\left(w+\left(\tau_{r}^{(m)}-\tau_{l}^{(m)}\right) / T_{c}\right) / \beta\right]^{(m)}}^{x^{(m)}}{ }^{\left(m+\left(\tau_{r}^{(m)}-\tau_{l}^{(m)}\right) / T_{c}\right.} .
\end{gathered}
$$

\section{BER Integral Form for Multiuser Chaos-Based DS-CDMA}

The overall BER of the user $m$ takes the following form:

$$
\begin{aligned}
\operatorname{BER}^{(m)}= & P\left(s_{i}^{(m)}=+1\right) P\left(\widehat{s}_{i}^{(m)}=-1 \mid s_{i}^{(m)}=+1\right) \\
& +P\left(s_{i}^{(m)}=-1\right) P\left(\widehat{s}_{i}^{(m)}=+1 \mid s_{i}^{(m)}=-1\right),
\end{aligned}
$$

where

$$
\begin{aligned}
P\left(\widehat{s}_{i}^{(m)}=-1 \mid s_{i}^{(m)}=+1\right) & =P\left(D_{i}^{(m)}<0 \mid s_{i}^{(m)}=+1\right) \\
& =P\left\{E_{b c}^{(i, m)}+\zeta_{i}^{(m)}+\psi_{i}^{(m)}<0\right\}, \\
P\left(\hat{s}_{i}^{(m)}=+1 \mid s_{i}^{(m)}=-1\right) & =P\left(D_{i}^{(m)}>0 \mid s_{i}^{(m)}=-1\right) \\
& =P\left\{-E_{b c}^{(i, m)}+\zeta_{i}^{(m)}+\psi_{i}^{(m)} \geq 0\right\} .
\end{aligned}
$$


In the following section, we will develop our approach to compute the bit error rate of the chaos-based DS-CDMA system.

4.1. BER Analysis Approach. In order to compute the bit error rate, the moments of first- and second-order of different noise sources must be computed.

In (15), the noise samples $n_{k}$ and chaotic samples $x_{k}^{(m)}$ are independent. After correlation with chaotic sequence, $\zeta_{i}^{(m)}$ is still a Gaussian random variable with zero mean and variance equal to

$$
\operatorname{Var}\left(\zeta_{i}^{(m)}\right)=\frac{N_{0}}{2} E_{b c}^{(i, m)}
$$

The Gaussian distribution of multiple-access interference has been examined in [39] when a binary spreading sequences of Markov chains are used. In our case, a real value chaotic sequence is used to spread the data symbols like in the chaos-based communication systems used in $[2,28-$ 30]. According to the central limit theorem, the sum of a large number of random variables from different chaotic sequences and from different delayed versions of the same chaotic sequence follow the Gaussian distribution [2, Section 3.2.3] and in $[29,30]$. Hence, $\gamma_{w, i}^{(m)}$ and $\alpha_{w, i}^{(m)}$ terms in (17) can be treated as Gaussian variables.

For (18), $\gamma_{w, i}^{(m)}$ is a zero mean Gaussian variable with (see Appendix A)

$$
\begin{gathered}
E\left[\gamma_{w, i}^{(m)}\right]=0, \\
\operatorname{Var}\left[\gamma_{w, i}^{(m)}\right]=E\left[\left|\gamma_{w, i}^{(m)}\right|^{2}\right] .
\end{gathered}
$$

According to the statistical properties of chaotic sequences mentioned in Appendix A, It can be easily demonstrated that the variance of $\gamma_{w, i}^{(m)}$ is

$$
\operatorname{Var}\left[\gamma_{w, i}^{(m)}\right]=\sum_{\substack{n=1 \\ n \neq m}}^{M} \sum_{l=0}^{L_{m}}\left|C_{l}^{(n)}\right|^{2} \sigma_{x}^{2} .
$$

Channel coefficients are normalized, and then variance becomes

$$
\operatorname{Var}\left[\gamma_{w, i}^{(m)}\right]=(M-1) \sigma_{x}^{2}
$$

where $\sigma_{x}^{2}$ is the mean power of the spreading chaotic sequence. It can be also demonstrated that the mean energy of a transmitted chaotic chip sequence $x_{k}$ is $E_{c}=T_{c} \sigma_{x}^{2}$. $\alpha_{w, i}^{(m)}$ in (19) is a Gaussian random sequence (see Appendix B) with

$$
\begin{gathered}
E\left[\alpha_{w, i}^{(m)}\right]=0, \\
\operatorname{Var}\left[\alpha_{w, i}^{(m)}\right]=\sum_{\substack{l=0 \\
l \neq r}}^{L_{m}}\left|C^{(m)}\right|^{2} \sigma_{x}^{2} .
\end{gathered}
$$

Finally, multiuser interference and self-interference are two independent zero mean Gaussian random variables with variances (see Appendices A and B):

$$
\begin{aligned}
& \operatorname{Var}\left[\operatorname{MUI}_{i}^{(m)}\right]=\underbrace{\sum_{r=0}^{L_{m}}\left|C_{r}^{(m)}\right|^{2}}_{=1} \underbrace{T_{c} \sum_{w=i \beta+k}^{i \beta+\beta-1}\left|x_{w}^{(m)}\right|^{2}}_{=E_{b c}^{(i m)}}(M-1) \underbrace{T_{c} \sigma_{x}^{2}}_{=E_{c}}, \\
& \operatorname{Var}\left[\mathrm{SI}_{i}^{(m)}\right]=\underbrace{T_{c} \sum_{w=i \beta+k}^{i \beta+\beta-1}\left|x_{w}^{(m)}\right|^{2}}_{=E_{b c}^{(i, m)}} \underbrace{\sum_{r=0}^{L_{m}}\left|C_{r}^{(m)}\right|^{2} \sum_{\substack{l=0 \\
l \neq r}}^{L_{m}}\left|C_{l}^{(m)}\right|^{2} \underbrace{T_{c} \sigma_{x}^{2}}_{=E_{c}} .}_{=1-\sum_{l=0}^{L_{m}}\left|C_{l}^{(m)}\right|^{4}}
\end{aligned}
$$

Using (28) and (29), $D_{i}^{(m)}$ can be considered as a normal random variable, with the following moments:

$$
\begin{gathered}
E\left[D_{i}^{(m)}\right]=E_{b c}^{(i, m)} s_{i}^{(m)}, \\
\operatorname{Var}\left[D_{i}^{(m)}\right]=E_{b c}^{(i, m)} \xi_{i}^{(m)},
\end{gathered}
$$

where

$$
\xi_{i}^{(m)}=\frac{N_{0}}{2}+(M-1) E_{c}+\left(1-\sum_{l=0}^{L_{m}}\left|C_{l}^{(m)}\right|^{4}\right) E_{c} .
$$

According to the fact that data symbols of user $m$ are equiprobably distributed on $\{+1,-1\}$ and by using (30) and (31), it comes that the error probability of symbol $i$ for user $m$ (with energy $E_{b c}^{(i, m)}$ ) is $P_{\mathrm{er}}^{(i, m)}$ :

$$
P_{\mathrm{er}}^{(i, m)}=Q\left(\sqrt{\frac{E_{b c}^{(i, m)}}{\xi_{i}^{(m)}}}\right)
$$

with $Q(x)=\int_{x}^{+\infty}(1 / \sqrt{2 \pi}) e^{\left(-u^{2} / 2\right)} d u$.

It appears that the system performance depends on the energy of the transmitted data symbol. Because of the nonperiodic nature of the chaotic signals, the transmitted bit energy using the chaos-based DS-CDMA systems varies from one bit to another for the same user $m$. The total BER will be evaluated by integrating $P_{\text {er }}$ over all possible values of the bit 


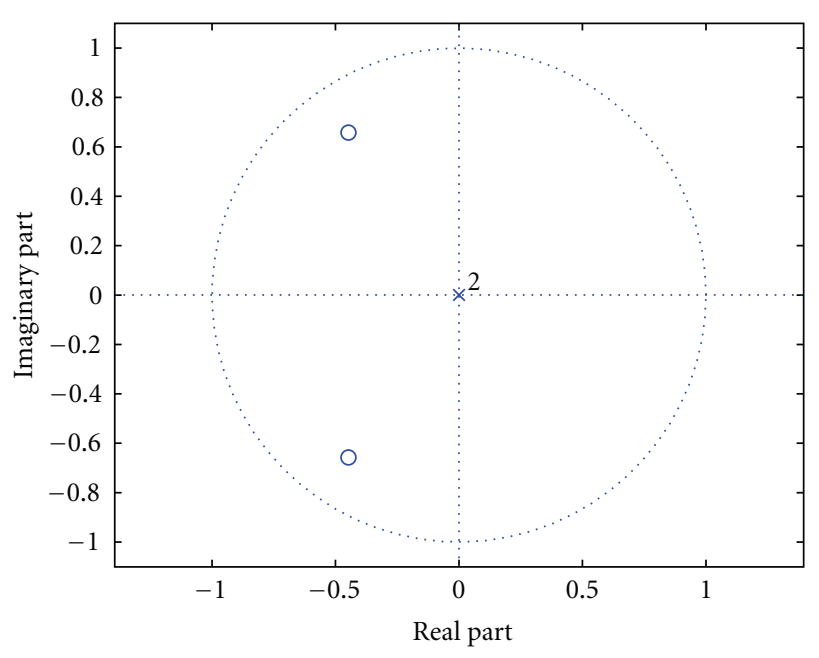

(a)
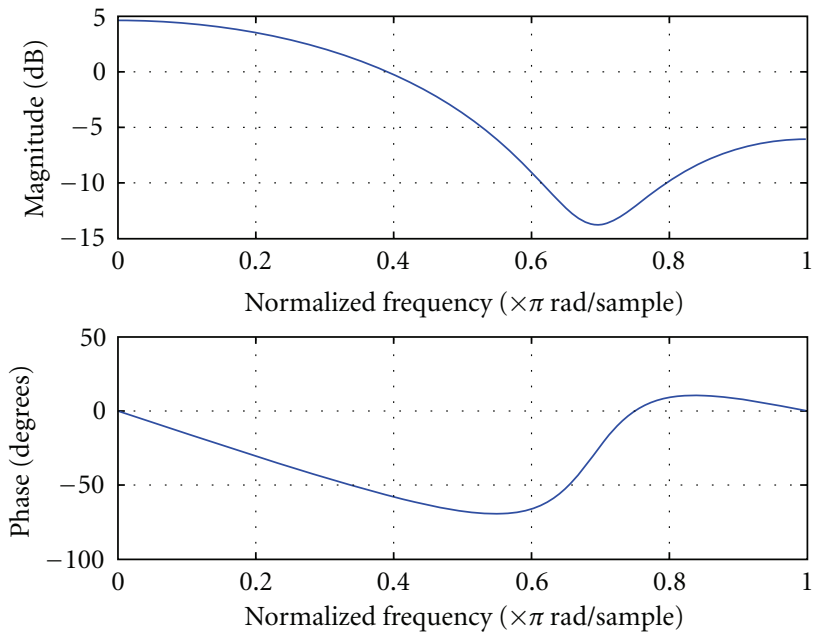

(b)

Figure 3: (a) Zeros of $H(z)$; (b) amplitude and phase responses of the channel.

energy $E_{b c}$. The total BER for user $m$ is expressed by

$$
\mathrm{BER}_{\text {chaos }}=\int_{0}^{+\infty} Q\left(\sqrt{\frac{E_{b c}}{\xi^{(m)}}}\right) p\left(E_{b c}\right) d E_{b c}
$$

\section{BER Computation}

In this section, we present two different methodologies to compute BER expression of (34).

5.1. Numerical BER Derivation. Following expression (34) it is necessary to get the bit energy distribution before computing the BER. Figure 2 gives the histogram of the bit energy for the PWL spreading sequence and for a spreading factor $\beta$ equal to 10 . The histogram of Figure 2 has been obtained using one million samples. From these samples, energies of successive bits are calculated for a given spreading factor. The bit energy is assumed to be the output of a stationary random process [40]; hence the histogram obtained in Figure 2 can be considered as a good estimation of the probability density function. When an analytical expression of the PDF is difficult to derive (example of PWL in Figure 2), the analytical integration of (34) seems intractable and the only way is to make a numerical integration. Using the histogram of Figure 2 we can compute the BER of (34) by using the following expression:

$$
\mathrm{BER}_{\mathrm{chaos}} \approx \sum_{i=1}^{c} Q\left(\sqrt{\frac{E_{b c}^{(i, m)}}{\xi_{i}^{(m)}}}\right) P\left(E_{b c}^{(i, m)}\right)
$$

where $c$ is the number of histogram classes and $P\left(E_{b c}^{(i, m)}\right)$ is the probability of having the energy in intervals centered on $E_{b c}^{(i, m)}$. This approach can be applied for any type of chaotic sequence with quite simple operations: histogram of the bit energy for a given spreading factor $\beta$ followed by a numerical integration. In addition, this approach explores the dynamics properties of chaotic sequence and gives results with very high accuracy.

In order to show the accuracy of our computing methodology, we compare simulation results together with the numerical integration method. For all the simulations, the number of users is taken arbitrary. In our simulations the number of users is fixed to $M=8$ for various spreading factor $\beta=20 ; 40 ; 80 ; 160 ; 320$. All chaotic sequences are normalized $\left(E\left(x^{(m)^{2}}\right)=1\right)$. In addition, all users use the same channel $h$ to transmit their signals. The impulse response of the channel used for transmission is

$$
h(t)=0.6742 \delta(t)+0.6030 \delta\left(t-T_{c}\right)+0.4264 \delta\left(t-2 T_{c}\right) .
$$

The characteristics of the channel are shown in Figure 3. It has two zeros inside the unit circle. The amplitude response of the channel presents a deep fading and the phase response is nonlinear.

Figure 4 gives simulation results together with the numerical integration method of the BER for various spreading factor plus the performance corresponding to the monouser BPSK case over an AWGN channel. This performance can be considered as a lower bound for the chaos-based DS-CDMA system because the transmitted bit energy is constant [31]. Computed BERs on Figure 4 are obtained by using the histograms of Figure 2 together with (35). The perfect match between simulation results and our numerical method confirms the accuracy of this approach based on the bit energy distribution. In addition, the good estimation of the BER for high $E_{b} / N_{0}$ confirms the validity of the Gaussian distribution of the multiuser and self interference noise.

For a DS-CDMA system low spreading factor has a limited benefit. For a low spreading factor $(\beta=20)$ 


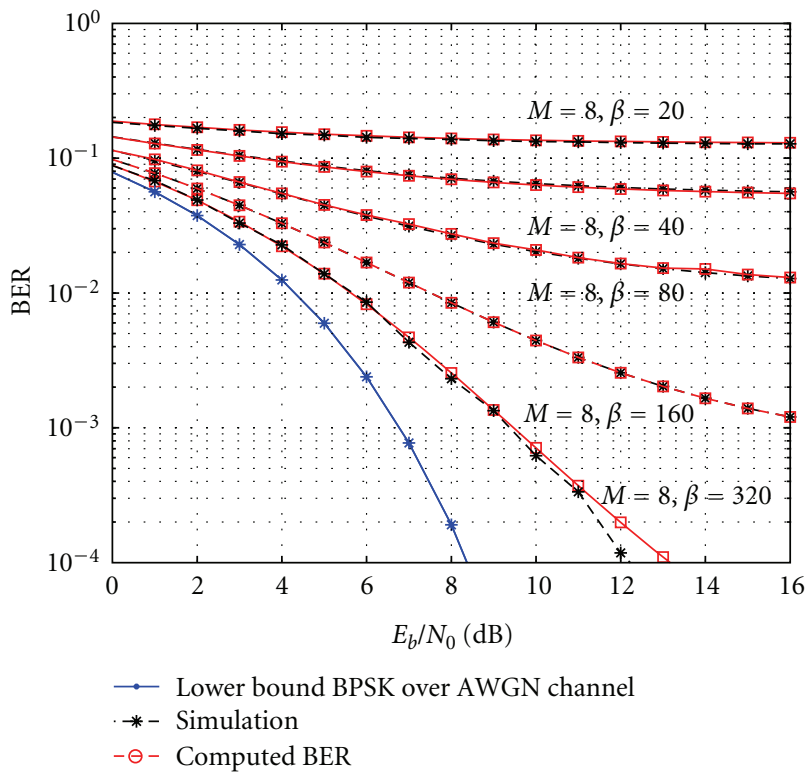

FIgURE 4: Numerical computation and simulated BER for $M=8$ and $\beta=20,40,80,160,320$.

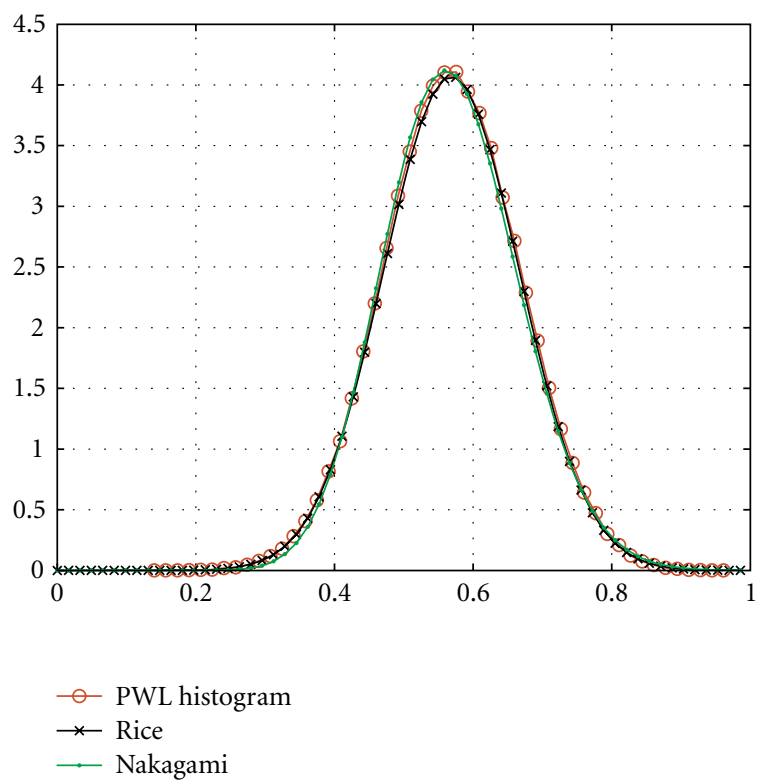

Figure 5: Approximation of the PDF associated to the PWL by a normalized root-square-energy histogram $(K=3, \phi=0.1, \beta=10)$.

multiuser interference is high, and the bit energy dispersion is large which implies a poor performances. To improve the performance of the system, it is necessary to increase the spreading factor. When we increase the spreading factor the dispersion of the bit is lower [31] and the multiuser interference is small.

5.2. Analytical BER Derivation. To get the analytical expression (34) it is necessary to have firstly the analytical expression of the PDF of the bit energy distribution and secondly to compute the integral of (34). Analytical expression of the PDF of (34) seems difficult to derive because chaotic samples are not totally independent.

In order to compute (34), two integral expressions of BER can be considered:

$$
\mathrm{BER}_{\text {chaos }}=\int_{0}^{+\infty} Q\left(\sqrt{\frac{2 V}{2 \xi_{i}^{(m)}}}\right) p(V) d V,
$$

where $V=E_{b c}^{(i, m)}$ and

$$
\mathrm{BER}_{\text {chaos }}=\int_{0}^{+\infty} Q\left(\sqrt{\frac{2 Y^{2}}{2 \xi_{i}^{(m)}}}\right) p(Y) d Y,
$$

where $Y=\sqrt{E_{b c}^{(i, m)}}$

Now, considering the expression (38) of BER, one can note that it has the same form than the expression of the BER obtained in the framework of mobile radio channels. Indeed, for a BPSK transmission on a radio channel with gain $\lambda$, the BER is expressed as

$$
\mathrm{BER}_{\substack{\text { Radio } \\ \text { channel }}}=\int_{0}^{+\infty} Q\left(\sqrt{\frac{2 \lambda^{2}}{2 \xi_{i}^{(m)}} E b}\right) p(\lambda) d \lambda .
$$

Closed form expressions are available for (39) in the case of channels following Rayleigh [41], Nakagami [42], or Rice distributions [43]. Since expression (38) is similar to expression (39), these previous results on (39) can be used for getting an analytical form of integral (38). Expression (37) does not allow to obtain a similar comparison, and to take advantage of existing integration results, one should then resort to numerical integration, as discussed in Section 5.1. The objective of this section consists of deriving an analytical expression of BER. Consequently, one will only focus on expression (38).

5.3. PDF Estimation of Root Square Energy. The PDF of the root square energy is fundamental in order to solve (38). The analytical derivation of the PDF seems intractable because of the difficulty to solve correlation integral. One proposes then to approximate this distribution by one of the three distributions (Rayleigh, Rice, and Nakagami) which will allow us to make use of the existing results on the computation of (39) to derive a closed-form expression of (38). This approximation has been investigated by plotting the histogram of the variable $Y$. The PDF shape associated to the PWL in Figure 5 gets the possibility to test the two candidates: Rice and Nakagami PDF.

The histogram of the root square bit energy obtained for one million samples of the PWL sequence is shown in Figure 5. We have tried to fit it by classical PDF laws. Figure 5 shows Rice and Nakagami-estimated PDF. The ChiSquare Goodness-of-Fit test confirms the fit for the two laws. Nevertheless, Chi-Square test gives priority to the Rice distribution (see Figure 6). 


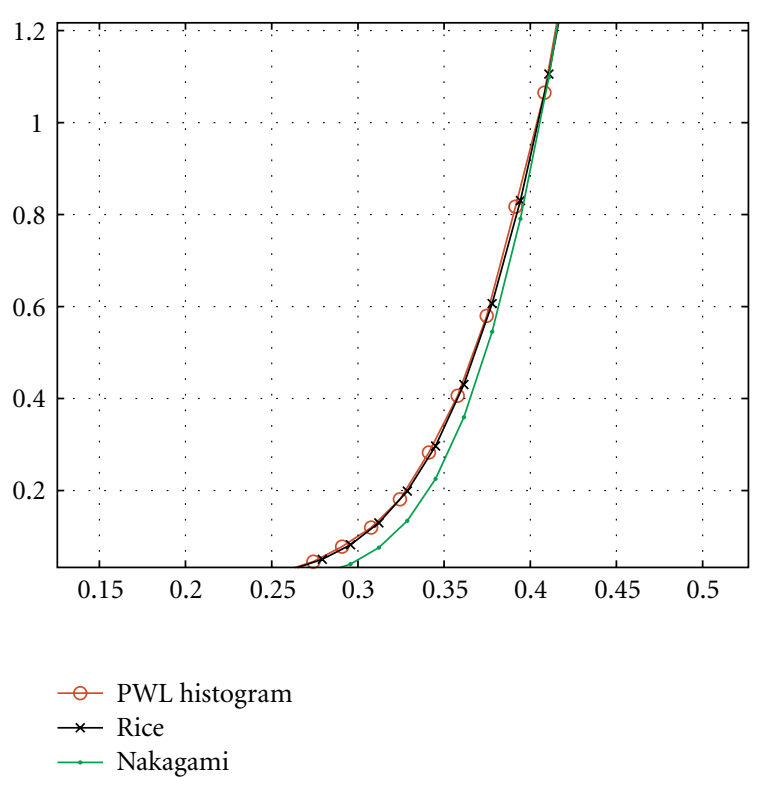

Figure 6: Zoom of the PDFs in the Figure 5.

\section{Analytical BER Calculation}

This section provides an expression of the BER by approximating the PDF energy with the Rice distribution.

6.1. Rice Distribution Parameters. $R=\sqrt{E_{b c}^{(i, m)}}$ is a random variable following a Rice distribution $P_{R}(r)$. The General Rice distribution function is defined in Appendix C.1.

General Rice distribution parameters are given by [43]

$$
\Omega=E\left(R^{2}\right) ; \quad \gamma=\frac{\operatorname{Var}\left[R^{2}\right]}{(\Omega)^{2}} .
$$

6.2. Parameters Estimation of the Rice Distribution. To have an analytical BER expression, the parameters of the Rice distribution should be derived from the parameters of the PWL sequence. Parameters given in expression (40) of Rice distribution are given by

$$
R^{2}=E_{b c}^{(i, m)}=T_{c} \sum_{k=0}^{\beta-1}\left(x_{i \beta+k}^{(m)}\right)^{2}
$$

where $x_{i \beta+k}^{(m)}$ can be seen as a random signal uniformly distributed on the interval $[-1,+1]$ (see [38]). $x_{i \beta+k}^{(m)}$ has a zero mean and its variance is $1 / 3$. Then the scale parameter $\Omega$ is given by

$$
\Omega=E\left(R^{2}\right)=T_{c} \sum_{k=0}^{\beta-1} E\left(\left(x_{i \beta+k}^{(m)}\right)^{2}\right)=\frac{T_{c} \beta}{3} .
$$

Variance of $R^{2}$ is then

$$
V\left[R^{2}\right]=E\left(\left(R^{2}\right)^{2}\right)-\left(\frac{T_{c} \beta}{3}\right)^{2}
$$

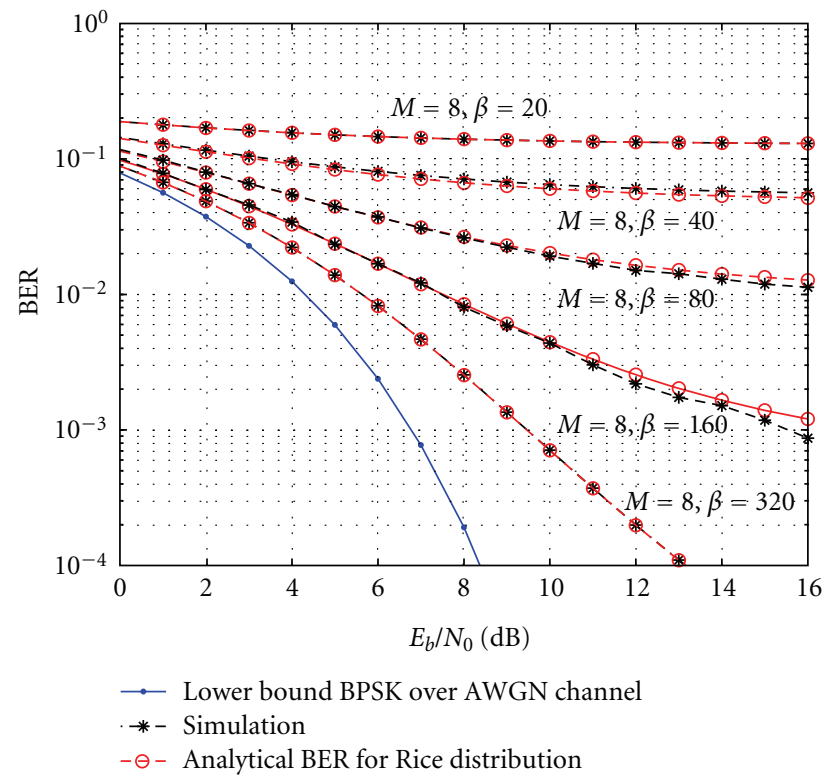

FIgURE 7: Simulated BER and analytical BER expression for Rice distribution for $M=8$ and $\beta=20,40,80,160,320$.

with

$$
E\left(\left(R^{2}\right)^{2}\right)=\frac{\beta T_{c}^{2}}{5}+2 T_{c}^{2} \sum_{n=1}^{\beta-1}(\beta-n) E\left(x_{i \beta+k}^{(m)}{ }^{2} x_{i \beta+k+n}^{(m)}{ }^{2}\right),
$$

where $E\left(x_{i \beta+k}^{(m)^{2}} x_{i \beta+k+n}^{(m)}{ }^{2}\right)$ is estimated using the PWL chaotic sequence. Then $\gamma$ can be obtained by (40) (Appendix C.1).

6.3. Analytical Expression of the BER. Following results of [43] the analytical BER is given:

$$
\begin{aligned}
& \operatorname{BER}_{P W L}(\beta) \\
& =Q(u, v)-\frac{1}{2}\left[1+\sqrt{\frac{d}{1+d}}\right] \exp \left(-\frac{u^{2}+v^{2}}{2}\right) I_{0}(u v),
\end{aligned}
$$

where the parameters $u, v, Q(\cdot, \cdot)$ and $I_{0}$ are given in Appendix C.2.

In simulations, the same parameters have been taken as before in the numerical integration. BER obtained using the analytical expression of (45) and the ones given by Monte Carlo simulations are compared in Figure 7. The lower bound BER of chaos-based communication system is also plotted for reference. It clearly appears in Figure 7 that we have a perfect match between simulations and the analytical results. Expression (45) can thus be used for all types of spreading factors even for very small ones.

\section{Conclusion}

In this paper we have proposed a new simple approach to compute BER for asynchronous chaos-based DS-CDMA systems over multipath channel. Because of the nonperiodic and the deterministic nature of chaotic sequence, 
the constant energy assumption or the standard Gaussian approximation of the decision variable in the output of correlator leads to inaccurate results in the BER expression. In our approach, neither the constant bit energy assumption, nor the Gaussian approximation for the decision variable is considered to compute the performance of the chaos-based communication system. The BER expression is computed in terms of the energy distribution, the number of paths, the noise variance, and the number of users. In order to study only the performance of the system, a perfect synchronization of the chaos is assumed, and we assume that channel coefficients and delay estimation are known in the receiver. The statistics of different noise sources were studied, and the means and variances were evaluated. The Gaussian distribution of the interfering noise is approximated thanks to the central limit theorem. Two methodologies are considered to derive the BER expression. Firstly, when the PDF of the bit energy has an irregular shape, only numerical integration method is possible in order to compute the BER. This numerical approach can be applied for a large chaosbased communication system and for any type of chaotic sequences independent from the initial condition of the sequence. Secondly, in special cases, when the distribution of the root square bit energy has a known distribution (Rice, Nakagami or Rayleigh), the analytical BER expression can be easily computed. For the PWL chaotic map, such an analytical expression has been obtained with perfect match with simulation. The analytical expression of BER for multiuser DS-CDMA over Rayleigh channel is under study.

\section{Appendices}

\section{A. Proof of (25) and (28)}

The term $\gamma_{w, i}^{(m)}$ is given by

$$
\left.\gamma_{w, i}^{(m)}=\sum_{\substack{n=1 \\ n \neq m}}^{M} \sum_{l=0}^{L_{n}} C_{l}^{(n)} s_{\left[\left(w+\left(\tau_{r}^{(m)}-\tau_{l}^{(n)}\right) / T_{c}\right) / \beta\right.}^{(n)}\right]_{w+\left(\tau_{r}^{(m)}-\tau_{l}^{(n)}\right) / T_{c}}^{x_{c}^{(n)}} .
$$

and so on $\gamma_{w, i}^{(m)}$ is the sum of different zero mean chaotic sequences. These chaotic sequences are obtained from different users and from different delayed version of the same chaotic sequence. Different chaotic sequences generated from the same generator with different initial conditions and the delayed version of the same chaotic sequence are uncorrelated $[1,29]$.

Because the mean of the chaotic sequences is equal to zero, the variance of $\gamma_{w, i}^{(m)}$ is

$$
\begin{gathered}
\operatorname{Var}\left[\gamma_{w, i}^{(m)}\right]=E\left(\left|\gamma_{w, i}^{(m)}\right|^{2}\right)-\underbrace{\left|E\left(\gamma_{w, i}^{(m)}\right)\right|^{2}}_{=0}, \\
\operatorname{Var}\left[\gamma_{w, i}^{(m)}\right]=E\left(\left|\gamma_{w, i}^{(m)}\right|^{2}\right),
\end{gathered}
$$

where



Because the chaotic samples of the PWL sequence have a very low correlation [38], it was proven in [29] that for one dimension recurrence chaotic map the mean function of the product of two chaotic samples denoted by $E\left[x_{k} x_{e}\right]$ in the case of $e=j+k$ for a positive integer $j$ is equal to zero. After developing the expression (A.3) the means of the terms having the form mentioned in (A.4) are equal to zero:

$$
\begin{aligned}
& E\left(C_{k}^{(n)} s_{\left\lfloor\left(w+\left(\tau_{r}^{(m)}-\tau_{k}^{(n)}\right) / T_{c}\right) / \beta\right.}^{(n)} x_{w+\left(\tau_{r}^{(m)}-\tau_{k}^{(n)}\right) / T_{c}}^{(n)}\right. \\
& \left.\quad \times C_{e}^{(n)} s_{\left\lfloor\left(w+\left(\tau_{r}^{(m)}-\tau_{e}^{(n)}\right) / T_{c}\right) / \beta\right.}^{(n)} x^{(n)}{ }_{w+\left(\tau_{r}^{(m)}-\tau_{e}^{(n)}\right) / T_{c}}^{(n)}\right)=0 .
\end{aligned}
$$

Since the chaotic sequences generated from a common generator with different initials conditions are uncorrelated, the terms having the form mentioned in (A.5) are also equal to zero:

$$
\begin{aligned}
& E\left(C_{k}^{(n)} s_{\left[\left(w+\left(\tau_{r}^{(m)}-\tau_{k}^{(n)}\right) / T_{c}\right) / \beta\right.}^{(n)}\right]^{x_{w+\left(\tau_{r}^{(m)}-\tau_{k}^{(n)}\right) / T_{c}}^{(n)}} \\
& \left.\quad \times\left. C_{j}^{(p)} s_{\left[\left(w+\left(\tau_{r}^{(m)}-\tau_{j}^{(p)}\right) / T_{c}\right) / \beta\right.}^{(p)}\right|^{x^{(p)}}{ }^{\left(\tau_{r}^{(m)}-\tau_{j}^{(p)}\right) / T_{c}}\right)=0 .
\end{aligned}
$$

Finally, only the following terms of (A.6) are not equal to zero:

$$
E\left(\left|C_{l}^{(n)}\right|^{2}\left(s_{\left[\left(w+\left(\tau_{r}^{(m)}-\tau_{l}^{(n)}\right) / T_{c}\right) / \beta\right.}^{(n)} x_{w+\left(\tau_{r}^{(m)}-\tau_{l}^{(n)}\right) / T_{c}}^{(n)}\right)^{2}\right) .
$$

After simplification, it can be easily demonstrated that the variance of $\gamma_{w, i}^{(m)}$ is

$$
\operatorname{Var}\left[\gamma_{w, i}^{(m)}\right]=\sum_{\substack{n=1 \\ n \neq m}}^{M} \sum_{l=0}^{L_{m}}\left|C_{l}^{(n)}\right|^{2} \sigma_{x}^{2} .
$$


For normalized channel coefficients the variance is

$$
\operatorname{Var}\left[\gamma_{w, i}^{(m)}\right]=(M-1) \sigma_{x}^{2} .
$$

For the global variance $\mathrm{MUI}_{i}^{(m)}$ of user $m$ is

$$
\operatorname{Var}\left[\mathrm{MUI}_{i}^{(m)}\right]=\operatorname{Var}(T_{c} \sum_{r=0}^{L_{m}} C_{r}^{(m)} * \sum_{w=i \beta}^{i \beta+\beta-1} \underbrace{x_{w}^{(m)}}_{A} \underbrace{\left[\gamma_{w, i}^{(m)}\right]}_{B}) .
$$

The terms $A$ and $B$ in the expression (A.9) are independent because the different chaotic sequences of different users are uncorrelated. For normalized channel coefficients the global variance $\mathrm{MUI}_{i}^{(m)}$ of user $m$ is:

$$
\operatorname{Var}\left[\operatorname{MUI}_{i}^{(m)}\right]=\underbrace{T_{c} \sum_{w=i \beta}^{i \beta+\beta-1}\left|x_{w}^{(m)}\right|^{2}}_{=E_{b c}^{(i, m)}}(M-1 \underbrace{T_{c} \sigma_{x}^{2}}_{=E_{c}}
$$

The variance $\mathrm{MUI}_{i}^{(m)}$ of the multiuser interference is:

$$
\operatorname{Var}\left[\operatorname{MUI}_{i}^{(m)}\right]=(M-1) E_{c} E_{b c}^{(i, m)}
$$

\section{B. Proof of (27) and (29)}

The term $\alpha_{w, i}^{(m)}$ is given by

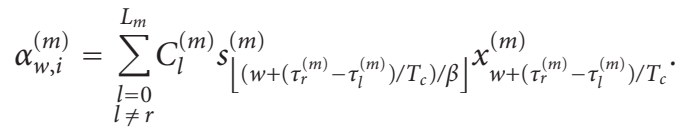

$\alpha_{w, i}^{(m)}$ is the sum of different delayed replicas of the same chaotic sequence multiplied by their correspondent channel coefficients, referring to the statistical properties mentioned in Appendix A and in $[29,38]$.

The mean $\alpha_{w, i}^{(m)}$ is equal to zero and the variance is

$$
\operatorname{Var}\left[\alpha_{w, i}^{(m)}\right]=E\left(\left|\alpha_{w, i}^{(m)}\right|^{2}\right)
$$

By developping the expression $E\left(\left|\alpha_{w, i}^{(m)}\right|^{2}\right)$ only the terms taking the forms $\left|C_{l}^{(m)}\right|^{2} E\left(\left|x_{w+\left(\tau_{r}^{(m)}-\tau_{l}^{(m)}\right) / T_{c}}^{(m)}\right|\right)$ are not equal to zero. We can easily prove that the variance of $\alpha_{w, i}^{(m)}$ is

$$
\operatorname{Var}\left[\alpha_{w, i}^{(m)}\right]=\sum_{\substack{l=0 \\ l \neq r}}^{L_{m}}\left|C_{l}^{(m)}\right|^{2} \sigma_{x}^{2}
$$

For the global variance $\mathrm{SI}_{i}^{(m)}$ we have:

$$
\operatorname{Var}\left[\mathrm{SI}_{i}^{(m)}\right]=\operatorname{Var}(T_{c} \sum_{r=0}^{L_{m}} C_{r}^{(m) *} \sum_{w=i \beta}^{i \beta+\beta-1} \underbrace{x_{w}^{(m)}}_{A} \underbrace{\left[\alpha_{w, i}^{(m)}\right]}_{B}) .
$$

The terms $A$ and $B$ in the expression (B.4) are independent because the different delayed versions of a chaotic sequence are uncorrelated. For normalized channel coefficients the global $\mathrm{SI}_{i}^{(m)}$ variance of user $m$ is

$$
\begin{gathered}
\operatorname{Var}\left[\mathrm{SI}_{i}^{(m)}\right]=T_{c}^{2} \sum_{w=i \beta}^{i \beta+\beta-1}\left|x_{w}^{(m)}\right|^{2} \sum_{r=0}^{L_{m}}\left|C_{r}^{(m)}\right|^{2} \sum_{\substack{l=0 \\
l \neq r}}^{L_{m}}\left|C_{l}^{(m)}\right|^{2} \sigma_{x}^{2}, \\
\operatorname{Var}\left[\mathrm{SI}_{i}^{(m)}\right]=E_{c} E_{b c}^{(i, m)} \underbrace{\sum_{r=0}^{L_{m}}\left|C_{r}^{(m)}\right|_{\substack{l=0 \\
l \neq r}}^{2} \sum_{D}^{L_{m}}\left|C_{l}^{(m)}\right|^{2}}_{D}
\end{gathered}
$$

In order to simplify the expression (B.6), the term $D$ can be expressed by

$$
\begin{aligned}
D= & {\left[\left|C_{o}^{(m)}\right|^{2}\left(\left|C_{1}^{(m)}\right|^{2}+\left|C_{2}^{(m)}\right|^{2}+\cdots+\left|C_{L_{m}}^{(m)}\right|^{2}\right)\right.} \\
& +\left|C_{1}^{(m)}\right|^{2}\left(\left|C_{0}^{(m)}\right|^{2}+\left|C_{2}^{(m)}\right|^{2}+\cdots+\left|C_{L_{m}}^{(m)}\right|^{2}\right) \\
& +\cdots \\
& \left.+\left|C_{L_{m}}^{(m)}\right|^{2}\left(\left|C_{0}^{(m)}\right|^{2}+\left|C_{1}^{(m)}\right|^{2}+\cdots+\left|C_{L_{m-1}}^{(m)}\right|^{2}\right)\right],
\end{aligned}
$$

where

$$
\begin{aligned}
D= & {\left[\left|C_{o}^{(m)}\right|^{2}\left(1-\left|C_{0}^{(m)}\right|^{2}\right)\right.} \\
& +\left|C_{1}^{(m)}\right|^{2}\left(1-\left|C_{1}^{(m)}\right|^{2}\right) \\
& +\cdots \\
& \left.+\left|C_{L_{m}}^{(m)}\right|^{2}\left(1-\left|C_{L_{m}}^{(m)}\right|^{2}\right)\right] \\
= & {[\underbrace{\left|C_{o}^{(m)}\right|^{2}+\left|C_{1}^{(m)}\right|^{2}+\cdots+\left|C_{L_{m}}^{(m)}\right|^{2}}_{=1}} \\
& \left.-\left|C_{o}^{(m)}\right|^{4}-\left|C_{1}^{(m)}\right|^{4}-\cdots-\left|C_{L_{m}}^{(m)}\right|^{4}\right] .
\end{aligned}
$$

Finally, the variance expression $\mathrm{SI}_{i}^{(m)}$ of user $m$ is

$$
\operatorname{Var}\left[\mathrm{SI}_{i}^{(m)}\right]=E_{c} E_{b c}^{(i, m)}\left(1-\sum_{l=0}^{L_{m}}\left|C_{l}^{(m)}\right|^{4}\right)
$$


C.

C.1. General Rice Distribution Function. The general Rice distribution is given by

$$
P_{R}(r)=\left\{\begin{array}{c}
\frac{2\left(K_{r}+1\right) r}{\Omega} \exp \left(-K_{r}-\frac{\left(K_{r}+1\right) r^{2}}{\Omega}\right) \\
\times I_{0}\left(2 \sqrt{\frac{K_{r}\left(K_{r}+1\right)}{\Omega}} r\right) \\
\text { if } r \geq 0 \\
0 \quad \text { if } r<0
\end{array}\right.
$$

where $r \geq 0, K_{r} \geq 0, \Omega \geq 0$

The parameters of Rice distribution are given by [43]

$$
\Omega=E\left(R^{2}\right) ; \quad \gamma=\frac{\operatorname{Var}\left[R^{2}\right]}{(\Omega)^{2}} .
$$

The shape parameter $K_{r}$ can be expressed in terms of $\gamma$ explicitly as [44]

$$
K_{r}=\frac{\sqrt{1-\gamma}}{1-\sqrt{1-\gamma}}
$$

By defining the new variables: $\sigma^{2}=\Omega / 2\left(K_{r}+1\right)$, and $\alpha^{2}=K_{r} \Omega /\left(K_{r}+1\right)$, the new PDF expression is obtained [44] by a simple change of variable in (C.1)

$$
p_{R}(r)= \begin{cases}\frac{r}{\sigma^{2}} \exp \left(-\frac{r^{2}+\alpha^{2}}{2 \sigma^{2}}\right) I_{0}\left(\frac{\alpha r}{\sigma^{2}}\right), & \text { if } r \geq 0, \\ 0, & \text { elsewhere. }\end{cases}
$$

C.2. The Parameters of BER over Rician Channel. The different parameters of the analytical BER expression of (45) are

$$
\begin{gathered}
u=\sqrt{\frac{\gamma_{r}^{2}[1+2 d-2 \sqrt{d(d+1)}]}{2(1+d)},} \\
v=\sqrt{\frac{\gamma_{r}^{2}[1+2 d+2 \sqrt{d(d+1)}]}{2(1+d)}}, \\
\sigma^{2}=\frac{\Omega}{2\left(K_{r}+1\right)}, \quad \alpha^{2}=\frac{K \Omega}{\left(K_{r}+1\right)}, \\
\gamma_{r}^{2}=\frac{\alpha^{2}}{2 \sigma^{2}}, \quad d=\sigma^{2} \frac{E b}{\xi^{(m)}},
\end{gathered}
$$

where $E b$ is the constant bit energy before spreading, $\xi^{(m)}$ is the sum of variances of different noises given in (32).

$Q(.,$.$) is the Marcum Q-function given by [43]$

$$
Q(\alpha, \beta)=\frac{1}{\alpha^{M-1}} \int_{\beta}^{\infty} x^{M} e^{-\left(x^{2}+\alpha^{2}\right) / 2} I_{M-1}(\alpha x) d x,
$$

and $I_{n}(x)$ is a modified Bessel function of the first kind.

\section{References}

[1] P. Stavroulakis, Chaos Applications in Telecommunications, CRC Press, New York, NY, USA, 2006.

[2] F. C. M. Lau and C. K. Tse, Chaos-Based Digital Communication Systems, Springer, New York, NY, USA, 2003.

[3] L. M. Pecora and T. L. Carroll, "Synchronization in chaotic systems," Physical Review Letters, vol. 64, no. 8, pp. 821-824, 1990.

[4] K. M. Cuomo, A. V. Oppenheim, and S. H. Strogatz, "Synchronization of lorenzbased chaotic circuits with aplication to communications," IEEE Transactions on Circuits and Systems, vol. 40, pp. 626-633, 1993.

[5] K. M. Cuomo and A. V. Oppenheim, "Circuit implementation of synchronized chaos with applications to communications," Physical Review Letters, vol. 71, no. 1, pp. 65-68, 1993.

[6] L. Kocarev and U. Parlitz, "General approach for chaotic synchronization with applications to communication," Physical Review Letters, vol. 74, no. 25, pp. 5028-5031, 1995.

[7] L. M. Pecora, T. L. Carroll, G. A. Johnson, D. J. Mar, and J. F. Heagy, "Fundamentals of synchronization in chaotic systems, concepts, and applications," Chaos, vol. 7, no. 4, pp. 520-543, 1997.

[8] U. Parlitz, L. O. Chua, L. Kocarev, K. S. Halle, and A. Shang, "Transmission of digital signals by chaotic synchronization," International Journal of Bifurcation and Chaos, vol. 2, pp. 973947, 1992.

[9] C. W. Wu and L. O. Chua, "Transmission of digital signals by chaotic synchronization," International Journal of Bifurcation and Chaos, vol. 3, pp. 1619-1627, 1993.

[10] S. Hayes and C. Grebogi, "Communication with chaos," Physical Review, vol. 71, pp. 3031-3034, 1993.

[11] U. Feldmann, M. Hasler, and W. Schwarz, "Communication by chaotic signals: the inverse system approach," International Journal of Circuit Theory and Applications, vol. 24, no. 5, pp. 551-579, 1996.

[12] G. Heidari-Bateni and C. D. McGillem, "Chaotic sequences for spread spectrum: an alternative to pn-sequences," in Proceedings of the IEEE International Conference on Selected Topics in Wireless Communications, pp. 437-440, Vancouver, Canada, June 1992.

[13] G. Heidari-bateni and C. D. McGillem, "Chaotic directsequence spread-spectrum communication system," IEEE Transactions on Communications, vol. 42, no. 2-4, pp. 15241527, 1994.

[14] R. L. Peterson, R. E. Zeimer, and D. E. Borth, Introduction to Spread Spectrum Communications, Prentice-Hall, Upper Saddle River, NJ, USA, 1995.

[15] G. Mazzini, G. Setti, and R. Rovatti, "Chaotic complex spreading sequences for asynchronous DS-CDMA-part I: system modeling and results," IEEE Transactions on Circuits and Systems I, vol. 44, no. 10, pp. 937-947, 1997.

[16] R. Rovatti, G. Setti, and G. Mazzini, "Chaotic complex spreading sequences for asynchronous ds-cdma-part II: some theoretical performance bounds," IEEE Transactions on Circuits and Systems I, vol. 45, no. 4, pp. 496-506, 1998.

[17] C.-C. Chen, K. Yao, K. Umeno, and E. Biglieri, "Design of spread-spectrum sequences using chaotic dynamical systems and ergodic theory," IEEE Transactions on Circuits and Systems I, vol. 48, no. 9, pp. 1110-1114, 2001.

[18] R. Rovatti, G. Setti, and G. Mazzini, "Toward sequence optimization for chaosbased asynchronous DS-CDMA systems," 
in Proceedings of the IEEE Global Telecommunications Conference (GLOBECOM '98), pp. 2174-2179, Sydney, Australia, 1998.

[19] G. Setti, R. Rovatti, and G. Mazzini, "Synchronization mechanism and optimization of spreading sequences in chaos-based DS-CDMA systems," IEICE Transactions on Fundamentals of Electronics, Communications and Computer Sciences, vol. E82A, no. 9, pp. 1737-1746, 1999.

[20] S. Vitali, R. Rovatti, and G. Setti, "On the performance of chaos-based multicode DS-CDMA systems," Circuits, Systems, and Signal Processing, vol. 24, no. 5, pp. 475-495, 2005.

[21] G. Kolumbán, G. K. Vizvari, W. Schwarz, and A. Abel, "Differential chaos shift keying: a robust coding for chaos communication," in Proceedings of the International Workshop on Nonlinear Dynamics of Electronic Systems, pp. 92-97, Seville, Spain, 1996.

[22] G. Kolumbán, M. P. Kenedy, and G. Kis, "Multilevel differential chaos shift keying," in Proceedings of the International Workshop on Nonlinear Dynamics of Electronic Systems, pp. 191-196, Moscow, Russia, 1997.

[23] H. Dedieu, M. P. Kennedy, and M. Hasler, "Chaos shift keying: modulation and demodulation of a chaotic carrier using selfsynchronization chua's circuit," IEEE Transactions on Circuits and Systems, vol. 40, pp. 634-642, 1993.

[24] F. C. M. Lau and C. K. Tse, "Optimum correlator-type receiver design for CSK communication systems," International Journal of Bifurcation and Chaos, vol. 12, no. 5, pp. 1029-1038, 2002.

[25] P. Chargé and D. Fournier-Prunaret, "An m-ary orthogonalCSK communication system," in Proceedings of the International Symposium on Nonlinear Theory and Its Applications, Bologna, Italy, 2006.

[26] S. Azou, C. Pistre, L. L. Duff, and G. Burel, "Sea trial results of a chaotic directsequence spread spectrum underwater communication system," in Proceedings of the IEEE Oceans, pp. 1539-1546, San Diego, Calif, USA, 2003.

[27] G. Mazzini, R. Rovatti, and G. Setti, "Chaos-based asynchronous DS-CDMA systems and enhanced rake receivers: measuring the improvements," IEEE Transactions on Circuits and Systems I, vol. 48, no. 12, pp. 1445-1453, 2001.

[28] W. M. Tam, F. C. M. Lau, C. K. Tse, and M. M. Yip, "An approach to calculating the bit-error rate of a coherent chaosshift-keying digital communication system under a noisy multiuser environment," IEEE Transactions on Circuits and Systems I, vol. 49, no. 2, pp. 210-223, 2002.

[29] W. M. Tam, F. C. M. Lau, C. K. Tse, and A. J. Lawrance, "Exact analytical bit error rates for multiple access chaosbased communication systems," IEEE Transactions on Circuits and Systems II, vol. 51, no. 9, pp. 473-481, 2004.

[30] A. J. Lawrance and G. Ohama, "Exact calculation of bit error rates in communication systems with chaotic modulation," IEEE Transactions on Circuits and Systems I, vol. 50, no. 11, pp. 1391-1400, 2003.

[31] G. Kaddoum, P. Chargé, D. Roviras, and D. Fournier-Prunaret, "Comparaison of chaotic sequences in a chaos based DSCDMA system," in Proceedings of the International Symposium on Nonlinear Theory and Its Applications, Vancouver, Canada, 2007.

[32] G. Kaddoum, D. Roviras, P. Chargé, and D. Fournier-Prunaret, "Analytical calculation of BER in communication systems using a piecewise linear chaotic map," in Proceedings of the European Conference on Circuit Theory and Design, Seville, Spain, 2007.
[33] G. Kaddoum, P. Chargé, D. Roviras, and D. Fournier-Prunaret, "Analytical BER expression of multi-user chaos-based DSCDMA system using a piecewise linear chaotic map," in Proceedings of the International Symposium on Nonlinear Theory and Its Applications, Budapest, Hungary, 2008.

[34] G. Kaddoum, P. Chargé, D. Roviras, and D. Fournier-Prunaret, "A methodology for bit error rate prediction in chaosbased communication systems," Circuits, Systems, and Signal Processing, vol. 28, no. 5.

[35] G. Kaddoum, D. Roviras, P. Chargé, and D. Fournier-Prunaret, "Performance of multi-user chaos-based DS-CDMA system over multipath channel," in Proceedings of the International Conference on Signals, Circuits and Systems, Taipei, Taiwan, 2009.

[36] R. Rovatti, G. Mazzini, and G. Setti, "Enhanced rake receivers for chaos-based DS-CDMA," IEEE Transactions on Circuits and Systems I, vol. 48, no. 7, pp. 818-829, 2001.

[37] G. Kaddoum, D. Roviras, P. Chargé, and D. Fournier-Prunaret, "Robust synchronization for asynchronous multi-user chaosbased DS-CDMA," Signal Processing, vol. 89, no. 5, pp. 807818, 2009.

[38] P. Chargé, D. Fournier-Prunaret, and V. Guglielmi, "Features analysis of a parametric PWL chaotic map and its utilization for secure transmissions," Chaos, Solitons and Fractals, vol. 38, no. 5, pp. 1411-1422, 2008.

[39] H. Fujisaki and Y. Yamada, "On bit error probabilities of SSMA communication systems using spreading sequences of markov chains," IEICE Transactions on Fundamentals of Electronics, pp. 2669-2677, 2005.

[40] S. H. Isabelle and G. W. Wornell, "Statistical analysis and spectral estimation techniques for one-dimensional chaotic signals," IEEE Transactions on Signal Processing, vol. 45, no. 6, pp. 1495-1506, 1997.

[41] J. Cheng and N. C. Beaulieu, "Accurate DS-CDMA bit-error probability calculation in Rayleigh fading," IEEE Transactions on Wireless Communications, vol. 1, no. 1, pp. 3-14, 2002.

[42] R. Esposito, "Error probabilties for the nakagami channel," IEEE Transactions on Information Theory, vol. 13, no. 1, pp. 145-148, 1967.

[43] W. C. Lindsey, "Error probabilities for rician fading multichannel reception of binary and $\mathrm{N}$-ary signals," IEEE Transactions on Information Theory, vol. 32, pp. 339-350, 1964.

[44] A. Abdi, C. Tepedelenlioglu, M. Kaveh, and G. Giannakis, "On the estimation of the $\mathrm{K}$ parameter for the rice fading distribution," IEEE Communications Letters, vol. 5, no. 3, pp. 92-94, 2001. 